\title{
Prosthetic joint infections: what to do if the C-reactive protein level remains elevated
}

\author{
C. Ruef
}

Published online: 20 July 2010

(C) Urban \& Vogel 2010

As the number of implanted joint prostheses of various joints increases, so does the number of episodes of postoperative prosthetic joint infections. The management of such infections is difficult and usually requires a combined approach involving surgical debridement and long-term antibiotic therapy. With this approach, it is possible to retain the prosthesis in many cases, especially in those patients with early diagnosis of the infection $[1,2]$. If all goes well, antibiotic treatment can be stopped after 3 months for hip prostheses and 6 months for knee prostheses [1].

The C-reactive protein (CRP) level is routinely measured to assess the response to treatment in addition to clinical assessment. Typically, CRP values return to normal levels during antibiotic treatment. However, in some patients, CRP values may remain elevated. Such a situation usually raises concerns regarding the effectiveness of the chosen antibiotic treatment among treating clinicians. Concerns may even be large enough to result in either an exhaustive diagnostic investigation, at times involving several imaging methods, or the use of surgical exploration to rule out the persistence of infection. Whether or not an elevated CRP should result in an aggressive diagnostic workup is a matter of debate. Undoubtedly, elevated CRP values need a good clinical assessment of the patient, including good medical history and physical examination. In this issue of Infection, Piso and Elke publish their experience of 14 patients with prosthetic joint infections and persistently elevated CRP [3]. The authors adhered to the treatment protocol mentioned above, using surgical debridement and retention of the prosthesis in 11 patients,

C. Ruef ( $\square)$

University Hospital of Zurich, Zurich, Switzerland

e-mail: christian.ruef@usz.ch single-staged reimplantation in one patient, and two-staged reimplantation in two patients. They stopped the antibiotic treatment after the time points mentioned above, even if the CRP levels remained above the upper limit of normal. The patients were followed up for 2 years.

Interestingly, in most patients, the CRP value was lower after 2 years than during the treatment phase, but it did not reach normal levels in some patients. In one patient, there was a relapse of infection 5 months after stopping antibiotic treatment. In this case, the second pathogen was not identical to the first isolate. In all other patients, no recurrence of infection occurred within 24 months.

While this is a small study, the findings are, nevertheless, interesting. The study highlights that it is not justified launching a diagnostic workup in each patient with a persistently elevated CRP after an adequate duration of antimicrobial therapy if there are no other clinical signs for infection. Of course, this study does not provide the argument for abandoning CRP measurements or ignoring the results of such measurements. It just reminds us that CRP elevation may have a variety of causes, infection being only one of them.

Conflict of interest None.

\section{References}

1. Zimmerli W, Trampuz A, Ochsner PE. Prosthetic-joint infections. N Engl J Med 2004; 351: 1645-54.

2. Zimmerli W, Ochsner PE. Management of infection associated with prosthetic joints. Infection 2003; 31: 99-108.

3. Piso RJ, Elke R. Antibiotic treatment can be safely stopped in asymptomatic patients with prosthetic joint infections despite persistent elevated C-reactive protein values. Infection 2010; 38 (in press). doi:10.1007/s15010-010-0019-y. 